\title{
Die Landschaftsschutzplanung des Vierwaldstätterseegebietes
}

\section{Obersicht über das Planungsgebiet}

\subsection{Abgrenzung}

Das Planungsgebiet umfaßt die Landschaftsräume am See bis zur Horizontlinie, die meist durch die an den See stoßenden Voralpen- und Alpenketten markiert wird. Zusätzlich werden das ganze Rigimassiv, der Bürgenstock und der Lauerzersee in die Planung miteinbezogen. Aus Abb. 1 kann die Grenze des Planungsgebietes ersehen werden. Es umschließt die Luzern umgebenden Molasserücken des Dietschiberges, des Gütsch und der Biregg mit der Horwer-Halbinsel. Von Horw verläuft die Grenze über die Pilatusvorberge und das Pilatusmassiv bis zum Tomlishorn. Bei Alpnachstad wird das naturschutzwürdige Städerried nördlich des Flugplatzes Alpnach eingeschlossen. Dann bildet die Krete des Mueterschwanderberges die Abgrenzung. Die Kuppe des Rotzberges mit der Burgruine wird bis zur Kapelle Allweg (Franzosen-Einfall 1798) in Ennetmoos miteinbezogen. Von Allweg quert die Grenze zum Bürgenberg hinüber, folgt seinem Fuß und biegt am Ende des Flugplatzes Buochs zum Buochserhorn ab. Von dort markieren die Bergkulissen der Musenalp, der Schwalmis, des Oberbauenstockes und des Gitschen den Grenzverlauf. Der Brisengipfel und der Urirotstock gehören wohl zum Panorama des Vierwaltstättersees, sind aber vom Landschaftsraum des Sees abgesetzt. Bei Seedorf bilden das Schloß à Pro und das Kloster als Kulturobjekte zusammen mit dem Schilf- und Riedufer des Reußdeltas einen integrierenden Bestandteil der Seelandschaft. Ob Flüelen umschließt die Grenze die Eggberge und folgt weiter der Krete von Diepen und Rophaien, Huserstock und Fronalpstock nach Brunnen, zum Delta der Muota. Angrenzend an den Lauerzersee wurden der Sägel, die größte Riedlandschaft der Zentralschweiz und ein Teil des Goldauer Bergsturzes mit einigen markanten Rippen der Rigi miteinbezogen. Von Arth verläuft die Grenze durch den Zugersee und um die Hohle Gasse bei Immensee zum exponierten Chiemenhang und schließt das Planungsgebiet über den Meggerwald zum Dietschiberg bei Luzern ab.

\subsection{Bevölkerungsentwicklung und Tourismus}

In der folgenden Tabelle 1 der Einwohnerzahlen und Hotelbetten werden die Gemeinden herausgegriffen, deren Siedlungsschwerpunkt innerhalb der Planungsregion liegt:

In Tabelle 1 wurde versucht, die Vierwaltstätterseeregion nach See- und Bergabschnitten in Teilregionen zu gliedern. Beim Vergleich der Teilregionen ist aber zu beachten, daß sowohl der ganze See wie auch die betreffenden Bergabschnitte für den Fremdenverkehr aller Orte Anziehungspunkte bilden. Gesamthaft gesehen überragen Luzern und Umgebung als Zentrum des Fremdenverkehrs die andern Teilregionen, nämlich das Rigi-, das Urnersee-, das Bürgenstock-, das Brisen- und das Pilatusgebiet, in der Rangreihenfolge aufgezählt.

Als Gradmesser für touristische Erschließung der einzelnen Teilregionen diene die Zahl der Einwohner/ Hotelbett. Hier steht das Bürgenstockgebiet an der Spitze, wenn man von Ennetbürgen absieht, das vor allem als Wohnort dient. Ebenso ist das Rigigebiet mit 4,5 Einwohner/Hotelbett ein ausgeprägtes Fremdenverkehrsgebiet. Auffallend ist der Rückgang der Bevölkerung in Weggis von 1970-77. Er ist teilweise auf die Überalterung (16,9\% über 65 Jahre alt) und teilweise auf den Rückgang des Fremdenverkehrs und die Abwanderung von Gastarbeitern zurückzuführen. Über dem Durchschnitt von 10,2 Einwohner/Hotelbett der ganzen Region liegen das Umerseegebiet $(7,8)$, das Pilatusgebiet $(8,1)$ und das Brisengebiet $(9,8)$. Die Agglomeration Luzern bildet nicht nur das Zentrum des Fremdenverkehrs, sondern den gesamtwirtschaftlichen Schwerpunkt der Zentralschweiz. Die Einnahmen aus dem Tourismus wurden nach einer Gästebefragung des SCOPE-Institutes vom Juli bis September 1972 für die Stadt Luzern allein auf 120-130 Mio Franken geschätzt. 1972 erreichte die Zentralschweiz einen bisher nicht mehr erreichten Höhepunkt an Logiernächten.

Aus Tabelle 1 kann gut ersehen werden, daß der Tourismus in der Vierwaldstätterseeregion eine überragende Bedeutung einnimmt. Während sich die Hotellerie auf die erwähnten Orte konzentriert, bringt vor allem die Para-Hotellerie mit ihren Eigentumswohnungen und Ferienhäusern (1978: 55\% der Logiernächte in Hotels) eine starke Belastung der Landschaft. Auf diesen Gesichtspunkt weist auch KRIPPENDORF (1975): «Die Zukunft des Tourismus hängt weit mehr von

Dr. Richard Martin, Hünenbergstraße 31, 6006 Luzern. 


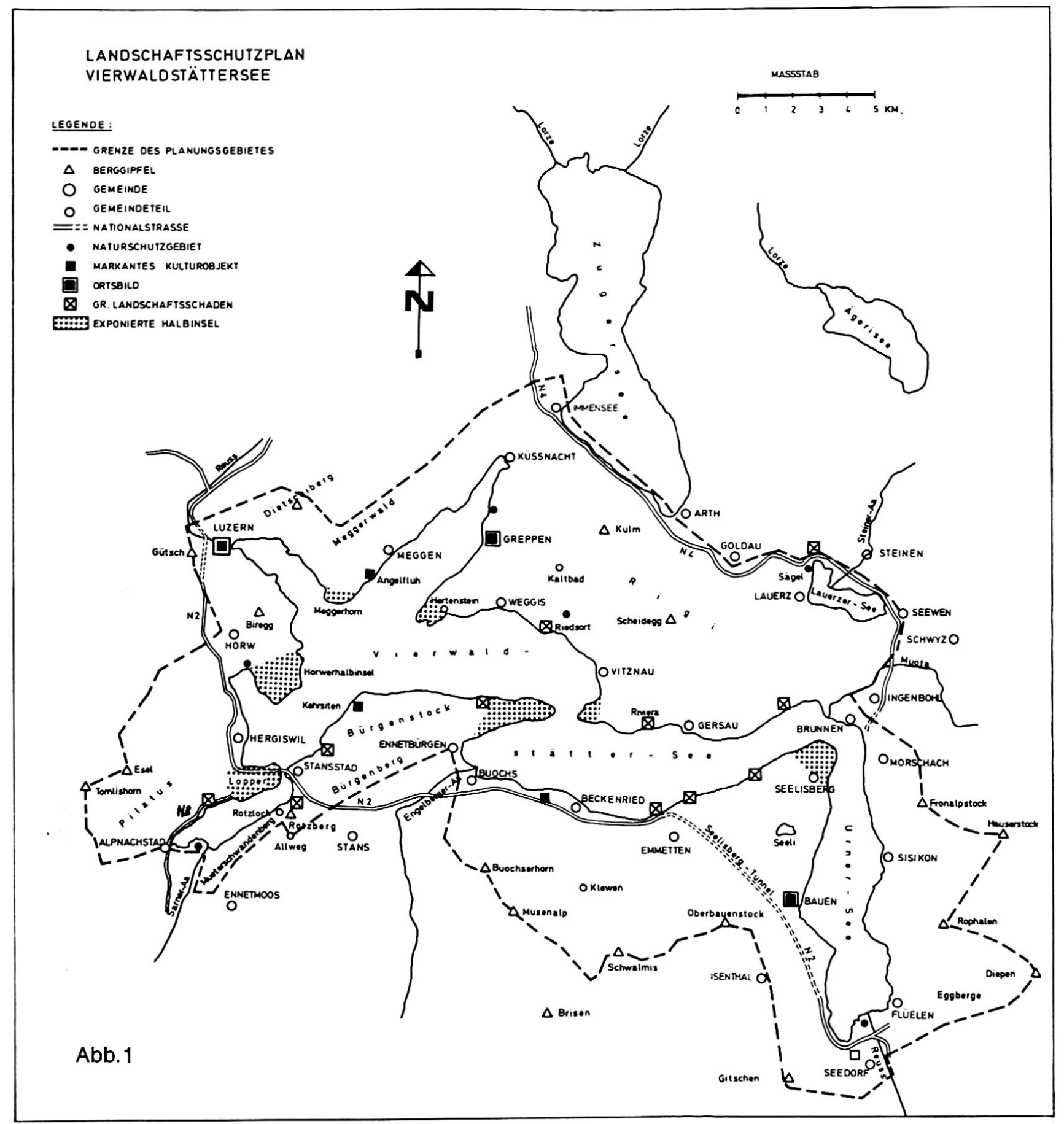

der Bewahrung der natürlichen Landschaft als von ihrer maximalen Erschließung ab». Die möglichst weitgehende Erhaltung der Vierwaldstätterseelandschaft ist das Ziel der Schutzplanung, auf die im folgenden eingegangen wird.

\section{Auftrag und Ziel der Landschaftsschutzplanung}

\subsection{Auftrag}

Bereits im Jahre 1967 hat die «Innerschweizer Regierungskonferenz» die «Interkantonale Kommission zum Schutze des Vierwaldstättersees» bestellt. Darin sind alle Anstößerkantone vertreten. Der Kommission wurde die Aufgabe übertragen, für den Vierwaldstättersee einen einheitlichen Schutzplan und eine Schutzverordnung zu entwerfen.

Im Jahre 1969 wurde der Firma Christian Stern, Büro für Garten- und Landschaftsgestaltung, Zürich, der Auftrag zur Aufnahme eines interkantonalen Schutzplanes erteilt. Schon 1968 war dieselbe Firma mit der Landschaftsschutzplanung im Kanton Luzern beauftragt worden. K. Holzhausen, dipl. Landschaftsarchitekt in der Firma Stern, hatte bereits am luzernischen Vierwaldstättersee zusammen mit dem Schreibenden 
Tab. 1: Bevölkerung und Hotelbetten

\begin{tabular}{lllll}
\hline $\begin{array}{l}\text { Region } \\
\text { und Gemeinde }\end{array}$ & $\begin{array}{l}\text { Ein- } \\
\text { wohner } \\
1970\end{array}$ & $\begin{array}{l}\text { Ein- } \\
\text { wohner } \\
31.12 .77\end{array}$ & $\begin{array}{l}\text { Hotel- } \\
\text { betten } \\
1979\end{array}$ & $\begin{array}{l}\text { Einw./ } \\
\text { Bett }\end{array}$ \\
\hline
\end{tabular}

\begin{tabular}{lrrrr} 
Luzern u. Umgebung & 84259 & 80392 & 5387 & 14,9 \\
Stadt Luzern & 69879 & 64081 & 5066 & \\
Horw LU & 10632 & 11539 & 183 & \\
Meggen LU & 3748 & 4772 & 138 & \\
& 13523 & 13489 & 2960 & 4,5 \\
Rigigebiet & 7956 & 8166 & 390 & \\
Küßnacht SZ & 367 & 321 & 20 & \\
Greppen LU & 2517 & 2270 & 1447 & \\
Weggis LU & & & & \\
(einschl. Rigi-Kaltbad) & 930 & 993 & 578 & \\
Vitznau LU & 1753 & 1739 & 385 & \\
Gersau SZ & & & 140 & \\
Rigi-Staffel u. -Kulm & & & & \\
& 4364 & 4507 & 555 & 8,1 \\
Pilatusgebiet & 4364 & 4507 & 382 & \\
Hergiswil & & & & \\
Alpnachstad und & & & 173 & \\
Pilatus-Kulm & & & & \\
& & & & \\
Bürgenstockgebiet & 4819 & 5840 & 954 & 6,1 \\
$\begin{array}{l}\text { Stansstad NW } \\
\text { (einschl. Kehrsiten und }\end{array}$ & 2469 & 3120 & 954 & 3,2 \\
Bürgenstock) & & & & \\
Ennetbürgen NW & 2350 & 2720 & - & \\
Brisengebiet & 5955 & 6655 & 670 & 9,9 \\
Buochs NW & 3232 & 3609 & 90 & \\
$\begin{array}{l}\text { Beckenried NW } \\
\text { (einschl. Klewenalp) }\end{array}$ & 2092 & 2279 & 500 & \\
Emmetten & 631 & 767 & 80 & \\
Urnerseegebiet & 9758 & 11199 & 1424 & 7,8 \\
Seelisberg UR & 584 & 604 & 170 & \\
$\begin{array}{l}\text { Bauen UR } \\
\text { Seedorf UR }\end{array}$ & 181 & 208 & 10 & \\
Flüelen UR & 1087 & 1295 & - & \\
Sisikon UR & 3750 & 1886 & 195 & \\
Morschach SZ & 703 & 762 & 135 & \\
Brunnen-Ingebohl SZ & 5111 & 6138 & 791 & \\
Total Planungsregion 112678 & 122082 & 11954 & 10,2 \\
\hline & & & & \\
\hline
\end{tabular}

die ersten Erfahrungen bei den Feldaufnahmen der Landschaftsschutzplanung gemacht. So konnten wir diese Arbeit nach der gleichen Methode fortführen, so daß die Schutzpläne des Kantons Luzern und des Vierwaldstätterseegebietes keine großen Unterschiede zeigen. In den Jahren 1970 und 1971 wurden die Feldaufnahmen für die Schutzplanung weitgehend abgeschlossen, und der Schutzplanentwurf im Maßstab 1:25000 lag im März 1972 vor.

\subsection{Ziel}

Ziel jedes Landschaftsschutzplanes ist es, alle jene $\mathrm{Ge}$ biete auszuscheiden, welche infolge ihrer landschaftlichen Einzigartigkeit und Erlebnisdichte nicht besiedelt, als Erholungsgebiete reserviert oder als Naturreservate geschützt werden sollen. Bei der Schutzplanung am Vierwaldstättersee geht es vor allem darum, die teilweise noch wenig berührte, voralpine See- und Gebirgslandschaft möglichst gut zu erhalten. Besondern Schutzes bedürfen die in den See vorragenden Halbinseln, wie z.B. Treib-Seelisberg und die gegen den See exponierten Steilhänge und Gipfelpartien der Voralpen (s. Abb. 1).

Rechtskräftige oder künftige Bauzonen in empfindlicher Lage sollen nur unter landschaftsschonenden Auflagen überbaut werden. Vor allem ist der Bestand an Ufergehölzen zu erhalten. Ebenso ist ein besonderes Augenmerk darauf zu richten, welche Seeuferpartien sich in Zukunft für öffentliche Zwecke eignen. Voralpen (s. Abb. 1).

\section{Methode des Vorgehens}

Zuerst wurden die vorhandenen Grundlagen wie Zonenpläne, GKP, Denkmalschutzverzeichnisse, Verkehrserschließungsprojekte, touristische Planungen usw. gesammelt und ausgewertet.

Bei den anschließenden Feldaufnahmen haben wir das ganze Gebiet begangen und befahren. Von Aussichtspunkten aus haben wir in gemeinsamer Diskussion eine begründete Abgrenzung der Schutzgebiete gesucht und auf der Landeskarte 1:25000 eingetragen. Die Inventarisierung der Einzelobjekte hat K. Holzhausen aus zeitlichen Gründen vorwiegend allein vorgenommen. Für jedes Schutzgebiet und Einzelobjekt wurde ein Aufnahme-Protokoll ausgefüllt. Die Grenzbereiche der Landschaftsschutz- und Schongebiete wurden auf farbige Lichtbilder und die Einzelobjekte auf Schwarzweißfotos aufgenommen.

In einer dritten Phase wurden die Planentwürfe mit den amtlichen Natur- und Heimatschutzkommissionen der Kantone Nidwalden, Schwyz und Uri besprochen, von der Interkantonalen Kommission zum Schutze des Vierwaldstättersees bereinigt, und den Instanzen der Sektion Natur- und Heimatschutz des Eidg. Oberforstinspektorates zur Orientierung vorgelegt.

Während der ganzen Planungsarbeit standen wir in engem Kontakt mit der Planungsstelle und dem Amt für Natur- und Heimatschutz des Kantons Luzern. Die letztere Amtsstelle übernahm auch die Sekretariatsarbeiten der Interkantonalen Kommission. 


\section{Inhalt des Landschaftsschutzplanes}

\subsection{Schutzgebiete}

Es wurden folgende Arten von Schutzgebieten kartiert:

\section{Naturschutzgebiete}

Es sind dies Gebiete von besonderem ökologischem und naturwissenschaftlichem Wert, wie Biotope seltener Tiere und Pflanzen. Am Vierwaldstättersee zählen dazu Uferriedflächen, zusammenhängende Schilfgürtel, Felsrippen usw. Naturschutzgebiete sollen durch ein Verbot von Bauten und andern Veränderungen umfassend geschützt werden.

Das größte vorauszusehende Naturschutzgebiet der Innerschweiz umfaßt das Sägelried, die angrenzenden Schilfgürtel am Lauerzersee und das benachbarte Bergsturzgebiet von Goldau. Zudem sollen das Seedorferried im Mündungsgebiet der Reuß und das Staderried bei der Mündung der Sarneraa als größere Naturschutzflächen ausgeschieden werden. Über das Steinibachried in der Horwer Seebuch hat der Kanton Luzern bereits eine Schutzverordnung erlassen.

\section{Uferstreifen}

Außerhalb der dicht bebauten Ortskerne wird ein Seeuferabstand von $25 \mathrm{~m}$ mit einem absoluten Bauverbot vorgeschlagen. Dadurch soll die Überbauung noch freier Uferpartien verhindert werden.

\section{Landschaftsschutzgebiete}

Es handelt sich dabei um noch ungestörte, typische oder einzigartige Landschaftsräume. Sie dienen in erster Linie als Erholungs- und Wandergebiete. Ebenso gliedern sie teilweise die Siedlungsräume und verhindern das Zusammenwachsen größerer Siedlungsbänder am See. In den Lanschaftsschutzgebieten sind landund forstwirtschaftliche Bauten weiterhin möglich. Hingegen soll auf jede ander Überbauung verzichtet werden.

Die Landschaftsschutzgebiete nehmen auf dem Entwurf des Schutzplanes den größten Raum ein. Sie seien daher im folgenden kurz angedeutet:

$\mathrm{Zu}$ den reizvollsten Seeufer-Partien der untern Seearme um den Kreuztrichter gehören die in den See vorspringenden Nagelfluhrippen des Meggenhorns und der Halbinseln von Kastanienbaum und Hertenstein. Hier wechseln bewaldete Felsvorsprünge der Molassehügel mit romantischen kleinen Buchten ab. Diese Halbinseln sind teilweise nur sehr locker mit Landsitzen überbaut. Einzig auf dem Stadtgebiet von Luzern ist der liebliche Charakter der Luzerner Bucht z.T. durch massive Überbauungen, vor allem südlich von Tribschen, gestört worden. Diese Überbauungsdichte darf nicht auf die Seeuferpartien der Gemeinden Horw und Meggen übergreifen. Die anmutige und sanfte Seelandschaft am Küßnachter- und Luzernersee, um den Kreuztrichter und an der Horwer Bucht trägt noch das Gepräge des Mittellandes.

Pilatus, Lopper, Bürgenstock und Rigi schließen die untern Seearme ab. Diese Voralpen bilden große zusammenhängende Landschaftsschutz- und Erholungsräume, die außerhalb der Siedlungen bis an die Seeufer hinunterreichen. Ihre steilen und bewaldeten Felsufer sind größtenteils nicht verbaut. Einzig an den sanftern Rigihängen zwischen Weggis und Vitznau sind größere Eingriffe durch allzu massive und heterogene Überbauungen erfolgt, so vor allem bei Riedsort.

Ähnlichen voralpinen Charakter zeigt das Gersauer Becken. Es wird durch die beiden Nasen von den untern Seearmen abgetrennt. Während die Bürgenstocknase nicht erschlossen und unberührt ist, wurden an der obern Nase auf Gersauer Gebiet Häuser als störende Bauten im Wald erstellt. Noch gravierender sind einzelne Überbauungen an den Steilhängen westlich von Gersau.

Der Urnersee trägt als fjordähnlicher Taltrog schon alpine Züge und konnte großenteils durchgehend von den Seeufern bis zur Gipfelregion als Landschaftsschutzgebiet vorgeschlagen werden. Die Steinschlaggalerien an der Axenstraße und eine Hochspannungsleitung über den Axenberg sind bis heute die auffallendsten Schönheitsfehler (Abb. 4).

Abdruck aus: Schweiz. Bauzeitung, a. a. $O$.

\section{Landschaftsschongebiete}

Darunter werden empfindliche Gebiete verstanden, welche für die Besiedlung in Frage kommen können, aber nur unter größter Schonung der Landschaft überbaut werden dürfen. Am Vierwaldstättersee sind es meist die gegen den See exponierten Steilhänge wie z.B. an der Rigi bei Gersau und Brunnen oder am Bürgenstock bei Kehrsiten. Aber auch Höhenkuppen wie die Klewenalp oder Rigi-Scheidegg sind durch Überbauung gefährdet.

Bei der Aufnahme der Landschaftsschutz- und Schongebiete haben wir auf die Ausdehnungsmöglichkeiten der einzelnen Gemeinden weitgehend Rücksicht genommen und um die Ortskerne nicht geschützte Gebiete offengelassen. Immer wieder sind wir in den Schutzgebieten auf das dringende Problem der Landschaftspflege gestoßen. Auf zahlreichen kleinen Bauerngütern an den Steilhängen ist das Existenzminimum der Bauern bei landwirtschaftlicher Nutzung in Frage gestellt. Als Alternative können aber auf keinen Fall alle diese Steilhänge mit Ferienhäusern überbaut werden. Durch die Ausrichtung von Beiträgen für bewirtschaftete Flächen muß eine Lösung dieses Problems gefunden werden.

\section{Ferienhausgebiete}

Um dem wachsenden Drang nach Zweitwohnungen entgegenzukommen, haben wir uns auch überlegt, wo neue geschlossene Ferienhaussiedlungen vom landschaftlichen Gesichtspunkt aus tragbar wären. Oft 
haben wir Ferienhausgebiete vorgeschlagen, wo bereits Bauabsichten oder Ansätze zu einer Überbauung vorhanden waren.

\section{Öffentliche Anlagen}

Bei unsern Feldaufnahmen haben wir zuhanden der Ortsplanungen angeregt, wo der öffentliche Seeuferansto $B$ in den einzelnen Gemeinden zur Schaffung von zusätzlichen Badeplätzen, Campingplätzen, Bootshäfen und Quaianlagen erweitert werden könnte.

\subsection{Schutzobjekte}

Folgende Arten von Einzelobjekten wurden in das Landschaftsschutzinventar aufgenommen:

\section{Aussichtspunkte}

Sie müssen gut erreichbar sein und eine besonders schöne Aussicht bieten, die nicht verbaut werden darf.

\section{Naturobjekte}

Dazu gehören Ufergehölze, Feldhecken, markante Baumgruppen und Einzelbäume, Felsrippen, erratische Blöcke, Wasserfälle usw. Sie bereichern das Landschaftsbild und sollten deshalb erhalten werden. Besondern Schutzes bedürfen die Ufergehölze. An einzelnen Uferpartien wurden sie leider teilweise widerrechtlich ausgeholzt, um aus Neubauten eine bessere Sicht auf den See zu erhalten. Vor allem auf der Horwer-Halbinsel sind in den letzten Jahren zusammenhängende Ufergehölze durch eine allzu large Rodungspraxis des Kreisforstamtes aufgelockert worden.

\section{Kulturobjekte}

Kirchen, Kapellen, besonders schöne Bauernhäuser und Speicher, Schlösser, Burgruinen, historische Stätten, Gebäudegruppen und Ortsbilder wurden kartiert. Bei der Auswahl von schützenswerten Kulturobjekten war vor allem der Gesichtspunkt des Landschaftsbildes maßgebend und weniger derjenige des kunsthistorischen Wertes.

\section{Wanderwege}

Hier wurden Anregungen gemacht, wo das Wanderwegnetz durch Ufer- oder Waldrandwege ergänzt werden sollte.

\section{Landschaftsschäden}

Am Vierwaldstättersee bilden die zahlreichen Steinbrüche empfindliche Eingriffe ins Landschaftsbild. Sie wurden als Landschaftsschäden inventarisiert. Auf Veranlassung des Eidg. Oberforstinspektorates hätte eine Detail-Inventarisierung der Steinbrüche die Grundlage der künftigen Abbauplanung bilden sollen. Dabei hätte man versucht, weniger exponierte Abbauvorkommen zu erschließen, um die seeseitigen Steinbrüche nach Möglichkeit aufzugeben und $\mathrm{zu}$ rekultivieren. Aus politischen und finanziellen Gründen haben die Innerschweizer Kantone mehrheit-

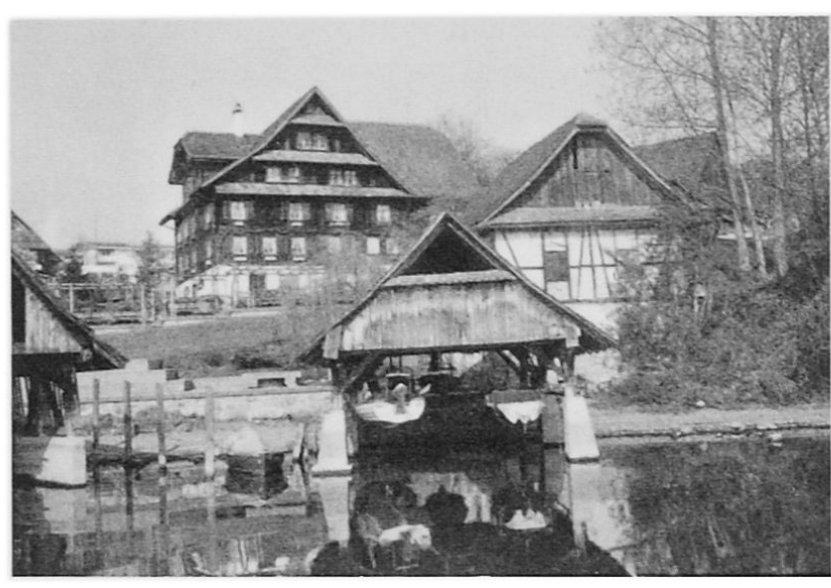

Abb. 2 Das «Fischerdörfchen» Angelfluh bei VorderMeggen, ein schützenswertes Ortsbild.

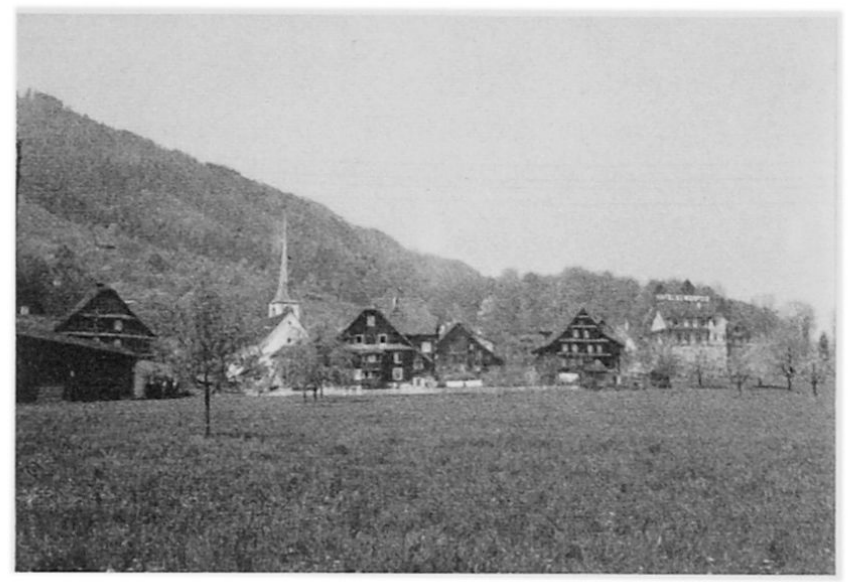

Abb. 3 Das schützenswerte Ortsbild von Greppen am Küßnachtersee.

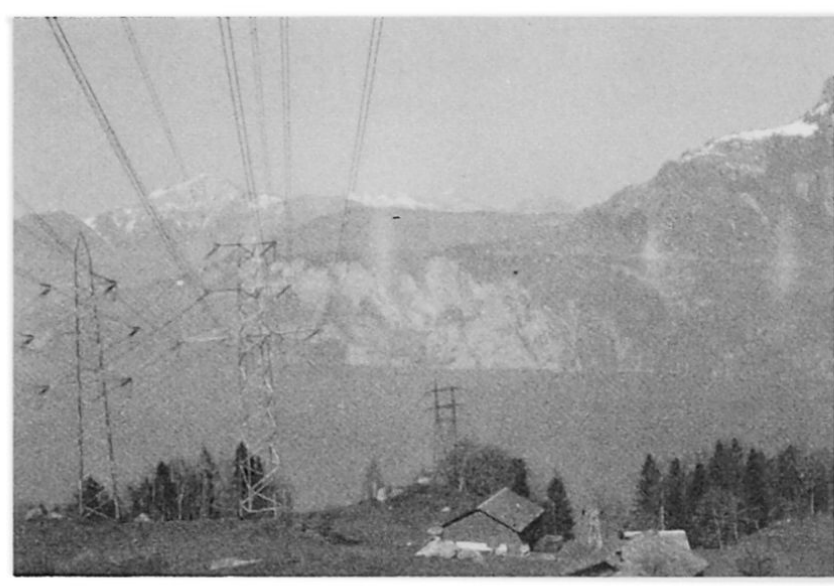

Abb. 4 Leitungsmasten stören die Landschaft auf Unter-Axen am Urnersee; im Mittelgrund Felsflühe des Seelisberg.

lich die Erstellung dieses geologischen Gutachtens abgelehnt und beschlossen, den Problembereich der Materialentnahme auf kantonaler Ebene zu regeln.

Immerhin konnte das Eidg. Oberforstinspektorat bei Rodungsgesuchen zur Erweiterung von Steinbrüchen landschaftspflegerische Auflagen machen. So wurde im Rotzloch, dem größten Steinbruch der Region, mit der Erteilung der Rodungsbewilligung ein Rekul- 
tivierungs- und Bepflanzungsplan durchgesetzt. Künftig muß in Terrassen abgebaut werden, die dem Schichtverlauf entsprechen. Die Abbauwände sind durch Gehölzgruppen wieder zu begrünen. Der Steinbruch Fallenbach an der Kantonsstraße zwischen Gersau und Brunnen, dessen Sprengungen und unerwartete Abbrüche sogar den Straßenverkehr gefährdeten, soll in 2-3 Jahren stillgelegt werden. Dabei muß ein Damm zum Schutz der Straße errichtet und aufgeforstet werden.

Ähnliche Eingriffe in die Seelandschaft bringen die Nationalstraßenbauten an der N2 zwischen Beckenried und Seedorf und an der N8 am Alpnachersee. Zwischen Beckenried und dem Nordportal des Seelisbergtunnels wird die $\mathrm{N} 2$ auf rund $3 \mathrm{~km}$ Länge über einen Lehnenviadukt geführt. Zur Auflockerung der horizontalen Viaduktlinie werden nach einem Bepflanzungskonzept einzelne Waldzipfel aufgeforstet. Beim Tunnelportal lie $\beta$ das betreffende Baukonsortium voreilig eine steile, bewaldete Felspartie ohne Bewilligung roden. Das Eidg. Oberforstinspektorat konnte nur noch über eine nachträgliche Rodungsbewilligung genaue Auflagen der Wiederaufforstung und Gestaltung des Tunnelportals machen. Andrerseits kann mit dem Aushub des Seelisbergtunnels der aufgegebene Steinbruch Rüttenen aufgefüllt und seine Böschung bepflanzt werden.

Soweit wir zu Gutachten oder Stellungnahmen betreffend Straßenprojekte aufgefordert wurden, haben wir versucht, dem Gesichtspunkt des Landschaftsschutzes Geltung zu verschaffen, so z. B. bei der Projektierung der Umfahrungsstraße von Vitznau (noch nicht begonnen) und bei der Führung der N 8 am Alpnachersee. Dort bilden die Felsverankerungen des Hanges beim künftigen Südportal des Loppertunnels einen unvorhergesehenen, starken Eingriff in das Uferbild. Ebenso wurden wir zur landschaftlichen Begutachtung der N4 zwischen Immensee und Seewen beigezogen und haben Empfehlungen zur bessern Einfügung der Nationalstraße in die Landschaft abgegeben. Beim Sägelried, wo heute die umstrittene Raststätte am Lauerzersee projektiert ist, haben wir seinerzeit an einer Begehung mit den Baubehörden von Bund und Kanton zur Schonung des Uferbiotops die Verlegung des Trassées in einen kurzen Tunnel verlangt. Leider wurde aus Kostengründen nicht darauf eingetreten.

\section{Realisierungsmöglichkeiten der Landschafts- schutzplanung}

\subsection{Vor Erlaß des Bundesbeschlusses}

Bereits während der Planaufnahmen suchten wir den Kontakt mit den Behörden der Gemeinden, deren Zonenplan rechtsgültig oder in Vorbereitung ist. Bei mehreren Gemeinden dienten unsere Schutzplan-Entwürfe als Grundlage für die Ortsplanung. In der Folge wurden wir bei zahlreichen Bauprojekten von Kantonsund Gemeindebehörden als Experten zugezogen.
Diese landschaftsschutzplanerischen Fachgutachten sowie Stellungnahmen zu den Zonenplanentwürfen haben eigentlich den Rahmen unseres Auftrages überschritten, waren aber als "Feuerwehrübungen» notwendig, damit nicht die Schutzbestrebungen laufend in Frage gestellt wurden, noch bevor deren Verwirklichung gesichert war.

\subsection{Die Auswirkungen des BMR}

Der Erlaß des Bundesbeschlusses über dringliche Maßnahmen auf dem Gebiete der Raumplanung vom 17. März 1972 (BMR) hat den Schutz der Vierwaldstätterseelandschaft entscheidend gefördert. Der Bundesbeschluß hat eine für alle Anstößerkantone einheitliche Rechtsgrundlage geschaffen. Gestützt auf den Bundesbeschluß erließ der Kanton Luzern am 23. Juni 1972 eine vorsorgliche Verfügung zum Schutze des luzernischen Vierwaldstättersees. Dadurch wurde der Entwurf des Schutzplanes für die luzernischen Vierwaldstättersee-Gemeinden rechtsverbindlich. Dann wurde der Schutzplanentwurf mit dem Regierungsrat des Kantons Nidwalden bereinigt und erhielt durch eine vorsorgliche Verfügung dieses Kantons im August 1972 Rechtskraft. Im Kanton Schwyz haben wir den Entwurf des Schutzplanes unter Führung des ehemaligen Regierungsrates Dr. $A$. $a b$ Yberg mit allen Gemeindebehörden des Planungsgebietes bereinigt. Der Kanton Schwyz erließ im November 1972 eine vorsorgliche Verfügung für die Gemeinden Küßnacht, Gersau und Ingenbohl (Brunnen). Diese vorsorglichen Verfügungen wurden erlassen, um die Bewilligungen für Bau'vorhaben zu verhindern, die dem Planungskonzept widersprechen, dies bevor der Bundesbeschluß rechtskräftig wurde.

Mit der Auflage der von Bern genehmigten Schutzpläne ist die Vierwaldstätterseelandschaft bis Ende 1979 provisorisch geschützt. Gegen die provisorischen Schutzpläne des BMR sind zahlreiche Einsprachen erhoben worden, so im luzernischen Vierwaldstätterseegebiet allein 454, die über 700 Einsprachepunkte betrafen. Einzelne Einsprecher, vor allem die Naturschutzorganisationen, verlangten die Verschärfung der Schutzmaßnahmen. Zur Behandlung der 1455 Einsprachen wurde im Kanton Luzern 1973 eine großrätliche Kommission bestellt, die dem Regierungsrat die Gutheißung oder Ablehnung der Einsprachen zu beantragen hatte. Diese «Kommission Landschaft» hat sich an Ort und Stelle von den Gemeindebehörden über die Situation der Einsprachen, die ErschlieBung der Grundstücke und die Ortsplanungstendenzen orientieren lassen. Am Vierwaldstättersee wurde der Seeuferschutz auf Bootsfahrten mit den Gemeindebehörden begutachtet und der Regionalplaner sowie einige Ortsplaner beigezogen. Bei der Beurteilung der Schutzwürdigkeit umstrittener Gebiete leisteten die Farbdias, die bei der Inventarisierung aufgenommen worden waren, eine wesentliche Entscheidungsgrundlage. Der Regierungsrat ist bei seinen Entscheiden 
meist den Anträgen der Kommission gefolgt und hat im ganzen Kanton rund 30\% der Einsprachen gutgeheißen. Dies bedingte meist jedoch nur geringfügige Grenzkorrekturen der Schutzgebiete. Andrerseits hat der Regierungsrat vor allem am Vierwaldstättersee auf Antrag der Kommission und im Falle der Horwer-Halbinsel nach eigenem Augenschein auf einer Bootsfahrt Planverschärfungen vorgenommen und zusätzliche Schutzgebiete ausgeschieden. Das Einspracheverfahren dauerte über 3 Jahre. Eine Reihe von Einsprechern rekurrierten gegen die Entscheide der Kantonsregierung an den Bundesrat, wurden jedoch meist abgewiesen. Wahrlich ein großer Aufwand unserer Planungsdemokratie!

Auch in den andern Kantonen hat das Einspracheverfahren nur geringfügige Änderungen der provisorischen Schutzpläne gebracht, so daß das Gesamtkonzept der Schutzplanung nicht beeinträchtig worden ist. Einzig im Kanton Schwyz brachte die Überbauung Riviera westlich von Gersau während der Gültigkeit des BMR einen neuen Eingriff. Dort hatte der Kanton den aufgelassenen Steinbruch mit schmalem Uferanstoß an ein Baukonsortium verkauft. Damit diese Überbauung auf dem Uferstreifen den gesetzlichen Grenzabstand zur Straße erhielt, mußte über die Uferlinie vorragend in den See hinaus gebaut werden. Zu diesem Zwecke verkaufte der Kanton den Bauherren einen Streifen Seegrund! Immerhin versuchte das zuständige Departement noch mit Hilfe des BMR das Bauvorhaben zu verhindern, indem es das Grundstück in den Seeuferabstand von $20 \mathrm{~m}$ miteinbezog. Dieses Bauverbot wurde aber vor Ablauf des BMR wieder aufgehoben. Heute riegeln zwei $40 \mathrm{~m}$ lange, zweigeschossige Flachdachbauten mit Ferienwohnungen und Bootsboxen das Ufer auf fast $100 \mathrm{~m}$ Länge ab. Häßliche Stützmauern müssen den dahinterliegenden Steinbruch sichern, wo jetzt die ARA von Gersau gebaut wird.

\subsection{Nach dem Ablauf des BMR}

Ende 1979 werden die Schutzgebiete des BMR ihre Rechtskraft verlieren. Das provisorische Bundesrecht muß durch kantonale oder kommunale Vorschriften abgelöst werden. So hat der Kanton Schwyz am 17.1.77 eine Verordnung zum Schutze der Seeufer erlassen und einen Streifen von $20 \mathrm{~m}$ an allen schwyzerischen Seeufern mit Bau- und Veränderungsverbot belegt. Vordringlich ist aber der Erlaß von kantonalen Naturschutzverordnungen, um die Uferriedlandschaften als Biotope zu sichern. Bis jetzt sind das Steinibachried bei Horw und das kleine Breitenacherried nördlich Greppen durch Verordnung geschützt. Das Sägelried im Kanton Schwyz wird im Rahmen der in Überarbeitung stehenden Schutzverordnung des Lauerzersees unter Schutz gestellt.

Die Landschaftsschutzgebiete am Vierwaldstättersee dürften erhalten bleiben, soweit sie im Rahmen von rechtskräftigen Ortsplanungen als Nichtbauland aus- geschieden worden sind. In einer Reihe von Gemeinden sind jedoch die Ortsplanungen noch nicht abgeschlossen, so in Küßnacht, Greppen, Weggis, Vitznau, Gersau, Brunnen-Ingenbohl und Lauerz, oder werden revidiert wie in Horw und der Stadt Luzern. Auch in Meggen ist die Seeuferplanung unterhalb der SBB-Linie noch nicht beendet. Deshalb wird im Kanton Luzern geprüft, ob allenfalls als Übergangsmaßnahme in den luzernischen Vierwaldstättersee-Gemeinden Planungszonen nach Artikel 27 und 36 des neuen Raumplanungsgesetzes erlassen werden sollen, um die Schutzmaßnahmen nach Ablauf des BMR bis zur Rechtsgültigkeit der Ortplanungen aufrecht zu erhalten. Im Kanton Nidwalden verfügen alle Seeuferorte wie auch Alpnach im Kanton Obwalden über rechtsgültige Zonenplanungen. Einzig in Stansstad ist eine Revision pendent, da in Kehrsiten provisorische Schutzgebiete des BMR ausgezont werden müssen. Um 1980 eine drohende Überbauung mit 35 Ferienhäusern am Fuße des Bürgenstocks vorläufig zu verhindern, hat die Gemeinde Stansstad für Kehrsiten im Dezember 1979 eine einjährige Bausperre erlassen. Im Kanton Uri haben die Gemeinden den kantonalen Landschaftsplan als Empfehlung in ihren Ortsplanungen großenteils integriert.

Es dürfte somit schwierig sein, den Auftrag der Interkantonalen Kommission zum Schutze des Vierwaldstättersees zu erfüllen und eine interkantonale Schutzverordnung zu erlassen. Auf jeden Fall muß verhindert werden, daß am Vierwaldstättersee und in andern Landschaften von nationaler Bedeutung die mit viel Mühe aufgebaute Schutzplanung einem neuen Bauboom zum Opfer fällt.

\section{Literaturangaben}

KASPAR, C.: Touristisches Leitbild Luzern, Schlußbericht, St. Gallen, 1973.

KRIPPENDORF, J.: Die Landschaftsfresser - Tourismus und Erholungslandschaft - Verderben oder Segen, Hallwag-Verlag, Bern, 1975.

MARTIN, R.: Grundlagenbericht Landschaft zum Leitbild der Raumordnung im Kanton Luzern, Raumplanungsamt des Kantons Luzern, 1977.

MARTIN, R.: Aspekte der Landschaftsschutzplanung des Vierwaldstättersees, Schweiz. Bauzeitung, 92. Jahrgang, Heft 37, Zürich, 1974.

Verkehrsverband Zentralschweiz: 32. Jahresbericht 1977, 33. Jahresbericht 1978, Hotelführer Zentralschweiz 1979.

\section{Résumé}

\section{Protection du paysage du lac des Quatre-Cantons}

Le plan de protection comprend tous les sites visibles depuis le lac, toute la région du Righi et le lac de Lauerz. 
En 1967, la Conférence des gouvernements de la Suisse centrale a institué la Commission intercantonale pour la protection du lac des Quatre-Cantons. Cette commission a été chargée d'élaborer un plan et un règlement de protection pour l'ensemble de la région.

En 1969, le bureau d'aménagement du paysage Stern, de Zurich, s'est vu confier l'établissement d'un plan intercantonal de protection. En sa qualité de géographe, l'auteur a été délégué par le canton de Lucerne pour collaborer à cette tâche. En 1970 et 1971, on procéda aux levés de terrain, et en 1972, on soumit le projet du plan aux autorités cantonales, qui y apportèrent quelques petites modifications. La méthode de travail et le contenu sont décrits aux chapitres 3 et 4 .

La réalisation de la protection du paysage a été accélérée par l'Arrêté fédéral du 17 mars 1972 instituant des mesures urgentes en matière d'aménagement du territoire. Pour délimiter les zones de protection exigées par cet arrêté, les autorités des quatre cantons se sont basées sur notre projet de plan de protection. Le plan intercantonal pour la protection du lac des Quatre-Cantons reste en vigueur jusqu'à fin 1979, date à laquelle l'arrêté fédéral doit être remplacé par la nouvelle loi fédérale sur l'aménagement du territoire. 